\title{
Erratum to: Industrial Internet of Things
}

\author{
Sabina Jeschke, Christian Brecher, Houbing Song \\ and Danda B. Rawat
}

\section{Erratum to:}

\section{S. Jeschke et al. (eds.), Industrial Internet of Things, Springer Series in Wireless Technology, DOI 10.1007/978-3-319-42559-7}

The book was inadvertently published without including the biographies of three editors in 'About the Editors' section. The erratum book has been updated with the correction.

The updated original online version for this book frontmatter can be found at DOI 10.1007/978-3-319-42559-7

\footnotetext{
S. Jeschke $(\bowtie) \cdot C$. Brecher

RWTH Aachen University, Aachen, Germany

e-mail: sabina.jeschke@ima-zlw-ifu.rwth-aachen.de

C. Brecher

e-mail: xcluster@wzl.rwth-aachen.de

H. Song

West Virginia University, Montgomery, WV, USA

e-mail: Houbing.Song@mail.wvu.edu

D.B. Rawat

Howard University, Washington, DC, USA

e-mail: db.rawat@ieee.org 